\title{
Role of miR-146a in human chondrocyte apoptosis in response to mechanical pressure injury in vitro
}

\author{
LEI JIN* , JIAN ZHAO*, WENSEN JING* ${ }^{*}$, SHIJU YAN, XIN WANG, CHUN XIAO and BAOAN MA \\ Department of Orthopedics, Tangdu Hospital, Fourth Military Medical University, Xi'an, Shaanxi 710038, P.R. China
}

Received April 21, 2014; Accepted June 11, 2014

DOI: $10.3892 / \mathrm{ijmm} .2014 .1808$

\begin{abstract}
MicroRNA (miR)-146a is known to be overexpressed in osteoarthritis (OA). However, the role of miR-146a in OA has not yet been fully elucidated. In the present study, we applied mechanical pressure of $10 \mathrm{MPa}$ to human chondrocytes for $60 \mathrm{~min}$ in order to investigate the expression of miR-146a and apoptosis following the mechanical pressure injury. Normal human chondrocytes were transfected with an miR146a mimic or an inhibitor to regulate miR-146a expression. Potential target genes of miR-146a were predicted using bioinformatics. Moreover, luciferase reporter assay confirmed that Smad4 was a direct target of miR-146a. The expression levels of miR-146a, Smad4 and vascular endothelial growth factor (VEGF) were quantified by quantitative reverse transcription PCR and/or western blot analysis. The effects of miR-146a on apoptosis were detected by Annexin V-fluorescein isothiocyanate (FITC)/propidium iodide (PI) flow cytometry. The results indicated that mechanical pressure affected chondrocyte viability and induced the early apoptosis of chondrocytes. Mechanical pressure injury increased the expression levels of miR-146a and VEGF and decreased the levels of Smad4 in the chondrocytes. In the human chondrocytes, the upregulation of miR-146a induced apoptosis, upregulated VEGF expression and downregulated Smad4 expression. In addition, the knockdown of miR-146a reduced cell apoptosis, upregulated Smad4 expression and downregulated VEGF expression. Smad4 was identified as a direct target of miR-146a by harboring a miR-146a binding sequence in the 3 '-untranslated region (3'-UTR) of its mRNA. Furthermore, the upregulation of VEGF induced by miR-146a was mediated by Smad4 in the chondrocytes subjected to mechanical pressure injury. These results demonstrated that miR-146a was overexpressed in
\end{abstract}

Correspondence to: Professor Baoan Ma, Department of Orthopedics, Tangdu Hospital, Fourth Military Medical University, No. 1 Xinsi Road, Xi'an, Shaanxi 710038, P.R. China

E-mail: gukemba@fmmu.edu.cn

*Contributed equally

Key words: apoptosis, osteoarthritis, mechanical injury, chondrocyte, miR-146a our chondrocyte model of experimentally induced human mechanical injury, accompanied by the upregulation of VEGF and the downregulation of Smad4 in vitro. Moreover, our data suggest that miR-146a is involved in human chondrocyte apoptosis in response to mechanical injury, and may contribute to the mechanical injury of chondrocytes, as well as to the pathogenesis of OA by increasing the levels of VEGF and damaging the transforming growth factor (TGF) $\beta$ signaling pathway through the targeted inhibition of Smad4 in cartilage.

\section{Introduction}

Osteoarthritis (OA) is the most common degenerative disease of the human articular cartilage, and a major cause of physical disability due to symptoms, such as pain, stiffness and loss of mobility, characterized by the progressive destruction of articular cartilage, subchondral bone alterations, the formation of osteophytes and synovitis (1). Although multiple patientspecific variables contribute to the risk of OA, such as aging, failure of nutrient supply and genetic predisposition, joint injuries increase the risk of developing OA by as much as 10-fold, or in some injuries, even by much more than 20 -fold (2). It has been suggested that excessive acute impact energy or chronic mechanical overload causes damage to chondrocytes and is thus responsible for OA (3). Yet, the mechanisms through which excessive mechanical force causes OA remain unknown. A recent study on cartilage injury in vitro demonstrated that chondrocyte damage and death caused by mechanical injury releases the effector molecules that activate chondroprogenitor cells in vitro that propogate and migrate to regions of damaged cartilage (4).

Another study revealed that these mechanically injured chondrocytes produce chemokines and cytokines that can cause joint inflammation and progressive cartilage loss (5). Blocking the expression of the effector molecules in mechanically injured joints may have the potential to prevent destructive inflammation. Therefore, the identification of the effector molecules and the molecular mechanisms responsible for regulating their expression is crucial for improving the effectiveness of current treatments for osteoarthritis.

Mechanical injury is a major cause of OA in humans. The pathological process of joint injury has 2 phases: the primary injury caused by a sudden mechanical force, and the secondary injury. The delayed, secondary injury is considered to be due to a cascade of biochemical reactions that are important in the 
mechanisms of OA. As cellular and micron-sized structural changes in vivo are hard to detect immediately post-injury, research on joint mechanical injury has focused on understanding the mechanisms of the secondary step that accounts for chondrocytes damaged by mechanical injury in vitro (6-8).

Apoptosis, or programmed cell death, is a physiological process responsible for maintaining homeostasis in articular cartilage (9). The OA cartilage contains a higher percentage of chondrocytes undergoing apoptosis than normal cartilage. Previous studies have demonstrated that chondrocyte apoptosis is related to the progression of OA. Chondrocytes undergo apoptosis in response to mechanical injury in vitro $(10,11)$. Chondrocyte apoptosis and necrosis have been reported in response to bovine and human cartilage wounding, following the mechanical injury loading of cartilage explants $(12,13)$. Mechanical injury as an important inducer of apoptosis plays a considerable role in the pathogenetic mechanisms of OA (11). It may be an important mediator of chronic articular lesions in OA.

MicroRNAs (miRNAs or miRs) are a family of 22 -nucleotide endogenous non-coding small RNAs that regulate the expression of multiple genes involved in a number of physiological functions and disease processes, including OA (14). Typically, they bind to the 3'-untranslated region (3'-UTR) of their target mRNAs and repress protein expression by affecting mRNA translation and/or destabilization (15).

Previous studies have indicated that miRNAs are known to play a key role in mediating the effects of the main risk factors for OA, such as innate and adaptive immune responses (16-18), aging, chronic pain and inflammation (19-22), through the control of target genes (23-25). The functions of regulated genes involve almost all aspects of cellular process, such as proliferation, differentiation, motivation, communication, senescence and apoptosis (26-28). Three previous studies (29-31) identified a signature of 17,16 and 7 miRNAs, respectively that distinguishes normal from osteoarthritic cartilage tissue, by microarray and real-time PCR assays. miR-146a is one of these identified miRNAs associated with OA cartilage (32).

Recent evidence suggests that miR-146a is markedly expressed in OA cartilage compared with normal cartilage, and its expression is induced by the stimulation of interleukin (IL)-1 $\beta$ (33). However, miR-146a expression levels in OA synovial tissue are low $(29,34)$. miRNA-146a expression is induced in response to lipopolysaccharide (LPS) and proinflammatory mediators in THP-1 cells and this induction is regulated by nuclear factor $(\mathrm{NF})-\kappa \mathrm{B}$, which in turn downregulates inflammatory cascades by decreasing the expression of IL-1 receptor-associated kinase-1 (IRAK1) and tumor necrosis factor (TNF) receptor-associated factor 6 (TRAF6). IRAK 1 and TRAF6 impair the NF- $\mathrm{NB}$ activation pathway and suppress the expression of NF- $\kappa \mathrm{B}$ target genes, such as IL-6, IL-8, IL-1 $\beta$ and TNF- $\alpha(16,23,25,35)$. These findings suggest that miR-146a plays a role in the damage of chondrocytes due to mechanical injury; miR-146a thus has potential as a novel therapeutic target in $\mathrm{OA}$.

In this study, we used a self-designed, mechanical pressurecontrolled cellular injury unit [utility model patent of China granted (36)] to precisely generate $10 \mathrm{MPa}$ of pressure on normal human chondrocytes inside a high-pressure container, which was placed in a $37^{\circ} \mathrm{C}$ thermostatic apparatus for $60 \mathrm{~min}$.
The results indicated that mechanical pressure affected the viability of the chondrocytes and induced the early apoptosis of chondrocytes. We used miRNA and gene microarray analysis to screen the differentially expressed genes and miRNAs between normal chondrocytes and mechanically injured chondrocytes. The results of the miRNA microarray and gene microarray demonstrated that the expression of mir-146a was upregulated in the mechanically injured chondrocytes. Bioinformatics analysis revealed that Smad4 is a potential target gene of miR-146a. Combined with the gene microarray report that the expression levels of Smad4 were downregulated and the expression levels of vascular endothelial growth factor (VEGF) were upregulated, we put forward the hypothesis that Smad4 regulates the expression of VEGF and mediates the damaging effects of miR-146a in the process of mechanical injury responsible for the pathogenesis of OA. We then evaluated the alterations in miR-146a, Smad4 and VEGF expression in normal chondrocytes following mechanical injury in vitro, and confirmed that Smad4 is a direct target of miR-146a. We found that in chondrocytes subjected to mechanical pressure injury, the expression levels of miR-146a and VEGF increased and the levels of Smad4 decreased.

Furthermore, we demonstrate that the increase in miR-146a expression downregulated Smad4 and upregulated VEGF expression, and induced the apoptosis of the mechanically injured chondrocytes. Conversely, the inhibition of miR-146a or the overexpression of Smad4 reduced VEGF expression in the mechanically injured chondrocytes. Taken together, these findings suggest that the dysregulation of miR-146a may contribute to the pathogenesis of OA by inhibiting Smad4, a key component in the anabolic forming growth factor (TGF)- $\beta$ pathway, by stimulating VEGF in the angiogenesis, chondrocyte hypertrophy and extracellular matrix degradation pathways, and by inducing chondrocyte death.

\section{Materials and methods}

Ethics statement. All human tissue samples were obtained in accordance with the World Medical Association Declaration of Helsinki Ethical Principles for Medical Research Involving Human Subjects, and were approved by the Ethics Review Committee of the Fourth Military Medical University, Xi'an, China (approval ID: 2013023) and written informed consent was obtained from all participating patients.

Isolation and culture of normal human chondrocytes. Normal knee cartilage samples were obtained from subjects undergoing post-traumatic above-knee amputation. Primary chondrocytes were isolated from the femoral condyles and tibial plateau of these human knee cartilage samples and cultured. The tissue samples were minced into small fragments, followed by digestion first with $0.25 \%$ trypsin (Gibco/Invitrogen, Carlsbad, CA, USA) for $30 \mathrm{~min}$ at $37^{\circ} \mathrm{C}$ and then with $0.2 \%$ collagenase (Sigma, St. Louis, MO, USA) for $5 \mathrm{~h}$ at $37^{\circ} \mathrm{C}$. The dissociated cell suspension was filtered through a $40-\mu \mathrm{m}$ cell strainer (BD Falcon, Bedford, MA, USA), and thecells were collected by centrifugation at $800 \mathrm{x} g$ for $10 \mathrm{~min}$. The chondrocytes were then cultured in DMEM/F-12 medium (Gibco/Invitrogen) supplemented with $10 \%$ fetal bovine serum (FBA, Gibco/ Invitrogen). Following overnight culture, non-adherent cells 
were removed, and the adherent cells were further incubated in fresh medium. Primary chondrocytes were cultured according to previously described methods (37-39). The chondrocytes used in the experiments were all first sub-culture cells. The results of quantitative reverse transcription PCR (RT-qPCR) demonstrated that all chondrocytes expressed type II collagen and did not express type I collagen.

Mechanical pressure injury to normal human chondrocytes. We used a self-designed, mechanical pressure-controlled cellular injury unit to precisely generate $10 \mathrm{MPa}$ of pressure on normal human chondrocytes which were grown to $70-80 \%$ confluence inside a high-pressure container, which was placed in a $37^{\circ} \mathrm{C}$ incubator for $60 \mathrm{~min}$. The control groups were placed in the unit but not loaded. This unit [utility model patent of China granted (36)], consists of 4 parts: the mechanical pressure container, the pressure control system, the temperature control system and the compressed gas cylinder (Fig. 1). The chondrocytes were cultured immediately following injury and maintained in culture for $8,12,24$ or $48 \mathrm{~h}$ to provide time for metabolic changes to occur before the subsequent experimental procedures.

MTT assay. The viability of the human chondrocytes was assessed at 8,12, 24 or $48 \mathrm{~h}$ following mechanical injury by MTT assay, as previously described (40). Human chondrocytes were seeded at a density of $1 \times 10^{5}$ cells/well in a 96-well plate and were grown to $70-80 \%$ confluence before mechanical pressure injury. The chondrocytes were cultured immediately after injury and maintained in culture for $4 \mathrm{~h}$ to provide time for metabolic changes to occur before the subsequent experimental procudures. Subsequently, each well was supplemented with $10 \mu \mathrm{l}$ of $5 \mathrm{mg} / \mathrm{ml} \mathrm{MTT}$ and incubated for an additional $4 \mathrm{~h}$ at $37^{\circ} \mathrm{C}$. The medium was then removed, and $150 \mu \mathrm{l}$ DMSO (Sigma-Aldrich, Shanghai, China) were added to solubilize the MTT formazan. The optical density was read with a spectrometer at a wavelength of $490 \mathrm{~nm}$. Wells without cells were used as blanks, and their values were considered the background values and subtracted from each sample.

Apoptosis assay by flow cytometry. The apoptotic rate of the chondrocytes was detected and quantified by flow cytometry following staining with Annexin V-FITC and propidium iodide (PI; both from Roche, Mannheim, Germany). The chondrocytes $\left(1 \times 10^{4}\right)$ were harvested, washed and incubated with Annexin V-PI for $15 \mathrm{~min}$ at room temperature in the dark. The chondrocytes were analyzed by flow cytometry using a Becton Dickinson fluorescence-activated cell sorter (BD Biosciences, San Jose, CA, USA) with emission filters of 530/30 nm (FITC) and 585/42 nm (PI). The percentage of apoptosis was analyzed using Becton Dickinson Cell Quest software. Each test was repeated in triplicate.

Microarray analysis. The miRNA expression profiles of the normal human chondrocytes and the human chondrocytes subjected to mechanical pressure injury were determined by miRNA microarray analysis using the GeneChip miRNA 3.0 Array (Affymetrix, Santa Clara, CA, USA), based on Sanger miRBase Release 17.0 (41). The transcription profiles of the normal human chondrocytes and the human chondrocytes
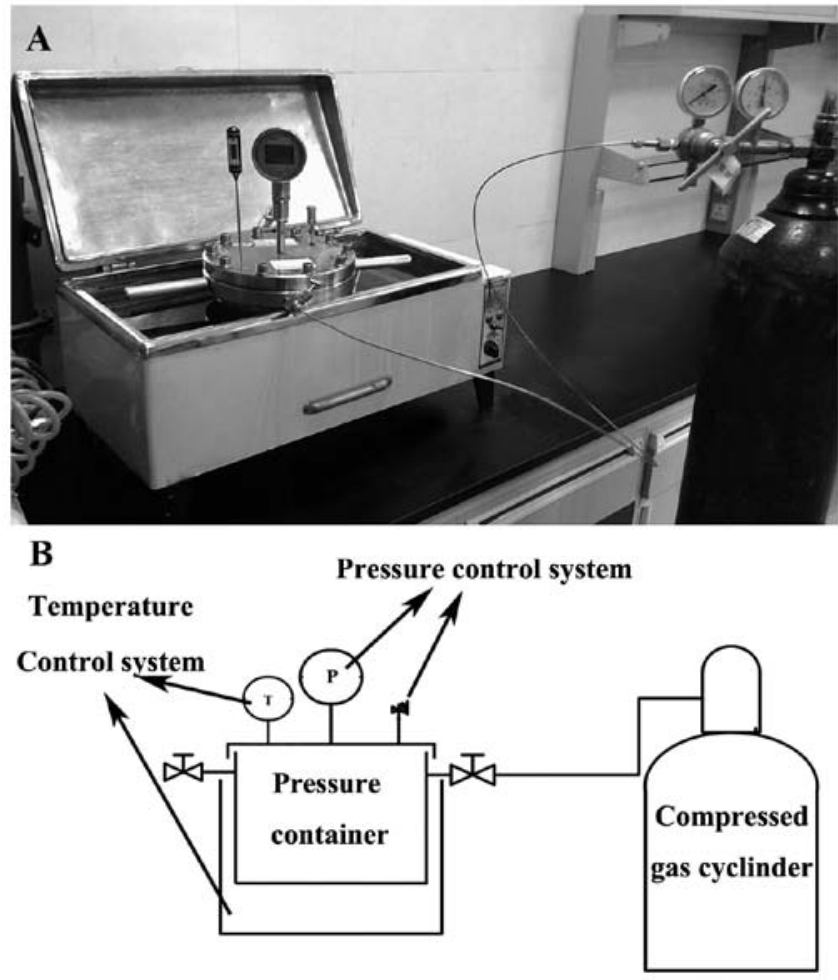

Figure 1. The device for applying pressure to cells. (A) The mechanical pressure-controlled cellular injury unit chart. (B) Schematic diagram of the custom-made instrument for applying mechanical pressure to chondrocytes. The main components of the instrument include a mechanical pressure container, a pressure control system, a temperature control system and a compressed gas cylinder.

subjected to mechanical pressure injury were determined by gene microarray analysis using the GeneChip Human Genome U133 Plus 2.0 Array (Affymetrix). The method was employed to identify differentially expressed miRNAs and genes between the normal chondrocytes and the chondrocytes subjected to mechanical pressure injury, as previously described $(23,31,42)$.

Target prediction. Putative target genes regulated by the miRNAs differentially expressed in normal chondrocytes and those subjected to mechanical pressure injury were predicted bioinformatically and combining the prediction of their supposed targets with the different genes expression of chondrocytes. Bioinformatics analysis was performed using these specific programs: miRanda (http://www.microrna.org), Pictar (http:// pictar.mdc-berlin.de/) and Targetscan (http://www.targetscan. org/), as previously described (31).

RNA oligonucleotides, plasmids, siRNA and transfection. The FAM modified 2'-O-me-oligonucleotides were synthesized by GenePharma (Shanghai, China). The sequences of the 2'-O-me-miR-146a mimics and 2'-O-me-miR-221 inhibitor, as well as a negative control of miRNA mimics (negative mimics) or inhibitors (negative inhibitors), were as follows: 5'-UGAGAACUGAAUUCCAUGGGUU-3', 5'-AACCCAUG GAAUUCAGUUCUCA-3' and 5'-UUGUACUACACAAAA GUACUG-3'. Smad4 siRNA (Smad4 siRNA: sc-29484; control siRNA: sc-37007) was purchased from Santa Cruz Biotechnology (Santa Cruz, CA, USA). When the cells were grown to 
$70-80 \%$ confluence, transfection was performed using the Lipofectamine $^{\mathrm{TM}} 2000$ transfection reagent (Invitrogen) according to the manufacturer's instructions. After $4 \mathrm{~h}$ of transfection, the medium was replaced with fresh medium (DMEM/F12) containing 10\% fetal bovine serum.

$R N A$ extraction and $R T-q P C R$. Total RNA (miRNA and mRNA) was extracted using TRIzol reagent (Invitrogen) according to the manufacturer's instructions. Subsequently, $1 \mu \mathrm{g}$ total RNA was reverse transcribed with a specific stem-loop primer for miRNA and with a random primer for mRNA using the AMV First-Strand cDNA Synthesis kit (Fermentas, Pittsburgh, PA, USA). After RT reaction, real-time PCR was performed on a Light Cycler 480 (Roche, Indianapolis, IN, USA) using ABI SYBR-Green PCR Master mix (Applied Biosystems, Bedford, MA, USA). $\beta$-actin and small nuclear RNA U6 were used as an internal normalized reference for CDNA and miRNA, respectively. The primers used were as follows: miR-146a forward, 5'-ACACTCCAGCTGGGTGAGAACTGAATTCC-3' and reverse, 5'-CTCAACTGGTGTCGTGGAGTCGGCAAT TCAGTTGAGAACCCATGG -3'; Smad4 forward, 5'-CTCT AAACCTCAGGCCACATC-3' and reverse, 5'-CAATACCT CCTCCATCAAAGC-3'; VEGF forward, 5'-ATGAACTTTC TGCTGTCTTGG-3' and reverse, 5'-TCACCGCCTCGGC TTGTCACA-3'; $\beta$-actin forward, 5'-CTCTTCCAGCCTT CCTTCCT-3' and reverse, 5'-TCATCGTACTCCTGCTT GCT-3'; U6 forward, 5'-CTCGCTTCGGCAGCACA-3' and reverse, 5'-AACGCTTCACGAATTTGCGT-3'. The RT-qPCR results were analyzed and expressed as the relative miRNA levels of the $\mathrm{Ct}$ (cycle threshold) value, which was then calculated to fold change by the value of each control sample set at 1 .

Western blot analysis. Chondrocyte total protein was washed with pre-chilled PBS and then subjected to whole-cell icecold lysis buffer with $50 \mathrm{mmol} / 1$ Tris- $\mathrm{HCl}, \mathrm{pH} 7.4 ; 1 \% \mathrm{NP}-40$; $150 \mathrm{mmol} / 1 \mathrm{NaCl} ; 0.1 \%$ sodium dodecyl sulfate (SDS); and supplemented with proteinase inhibitor (one tablet per $10 \mathrm{ml}$; Roche, Indianapolis, IN, USA). The concentration of proteins in the chondrocyte lysate was quantified using the DC protein assay kit (Bio-Rad Laboratories, Hercules, CA, USA), and diluted to an equal concentration with hypotonic buffer. A total of $40 \mu \mathrm{g}$ of the protein lysates was size-fractionated by 4-20\% SDS-PAGE and then transblotted electrically on to nitrocellulose membranes (Invitrogen). The membranes were blocked with TBST containing 5\% non-fat dry milk for $1 \mathrm{~h}$ and hybridized with primary antibody against Smad4 (1:1,000; Santa Cruz Biotechnology), VEGF (1:1,000; Santa Cruz Biotechnology), and GAPDH (1:5,000; Abcam, Shanghai, China) overnight at $4^{\circ} \mathrm{C}$. After washing 3 times with TBST, the membranes were hybridized with horseradish peroxidase (HRP)-conjugated anti-mouse or rabbit secondary antibody (Santa Cruz Biotechnology) for $2 \mathrm{~h}$. After washing 3 times with TBST again, the specific protein was detected by chemiluminescence using the enhanced chemiluminescence reagent, Pierce ECL Western Blotting Substrate (Thermo Fisher Scientific, Waltham, MA, USA). GAPDH was used as an internal control. The optical density of the immunoblots was quantified using Quantity One software (Bio-Rad Laboratories).
Luciferase reporter assay. The sequence of the human Smad4 3'-UTR containing the miR-146a binding side was amplified by PCR from genomic DNA using the following primers: forward, 5'-CCGCTCGAGTGAAGGAATCATTCCA GTGCTAG-3' and reverse, 5'-TGCTCTAGACTTGGTAAAA TTAACTCAC CCACA-3', and the PCR products were cloned into the pMIR-Report vector (Ambion, Austin, TX, USA) between the HindIII and SpeI sites downstream of the firefly luciferase gene present to develop the wild-type 3'-UTR luciferase reporter vector. The mutant 3'-UTR luciferase reporter vector was generated by site-directed mutagenesis using the QuikChange Mutagenesis kit (Stratagene, La Jolla, CA, USA) using the following primers: forward, 5'-TTAAA GGCAGAGA ACAAGAGAAAGTTAATTCACC-3' and reverse, 5'-GGTGAATTAACTTTCTCTTGTTCTCTGCC TTTAA-3'. All sequences of the amplified products were confirmed by DNA sequencing. Human chondrocytes were passaged on 24-wells plate the day prior to transfection to achieve $70-80 \%$ confluence on the following day. Human chondrocytes were transiently transfected using Lipofectamine 2000 (Invitrogen) according to the manufacturer's instructions, with wild-type or mutant-type pMIR-reportSmad4 vector in which the putative miR-146a binding site was mutated, and co-transfected with miR-146a mimics, miR-146a inhibitor or their negative control (NC) and inhibitor NC (GenePharma), individually. The human chondrocytes were also transfected with with Renilla luciferase reporter (pRL-TK) vector as an internal standard to determine and normalize the luciferase activity. At $24 \mathrm{~h}$ after transfection, the human chondrocyte lysates were extracted and luciferase activity was measured using a Dual Luciferase Reporter Assay System (Promega, Madison, WI, USA) on a Berthold AutoLumat LB9507 rack luminometer (Berthold Technologies China, Shanghai, China). The results were expressed as relative luciferase activity (firefly Luc/Renilla Luc). All experiments were repeated at least 3 times.

Statistical analysis. The results are expressed as the means \pm standard deviation unless otherwise indicated, and all error bars represent the standard deviation of the mean. Statistical analysis was carried out using the Student's t-test between 2 groups or one-way analysis of variance followed by Student-Newman-Kuels test for multiple comparisons with SPSS 13.0 statistical software (SPSS Inc, Chicago, IL, USA). A value of $\mathrm{P}<0.05$ was considered to indicate a statistically significant difference.

\section{Results}

Effects of mechanical pressure injury on chondrocyte viability. In a preliminary experiment, the human chondrocytes were exposed to 5, 8, 10 or $12 \mathrm{MPa}$ mechanical pressure for 10,30 or $60 \mathrm{~min}$, and chondrocyte viability was determined at 8 , 12, 24 or $48 \mathrm{~h}$ after the injury was sustained by MTT assay. The experiment demonstrated that loads below $8 \mathrm{MPa}$ did not result in any measurable cell death and loads above $12 \mathrm{MPa}$ resulted in extensive cell death. The OD values of the chondrocytes significantly decreased in a force-dependent manner at 8, 10, 12 Mpa after loading of mechanical pressure for $60 \mathrm{~min}$ $(\mathrm{P}<0.05)$ (Fig. 2A). The OD values of the chondrocytes signifi- 

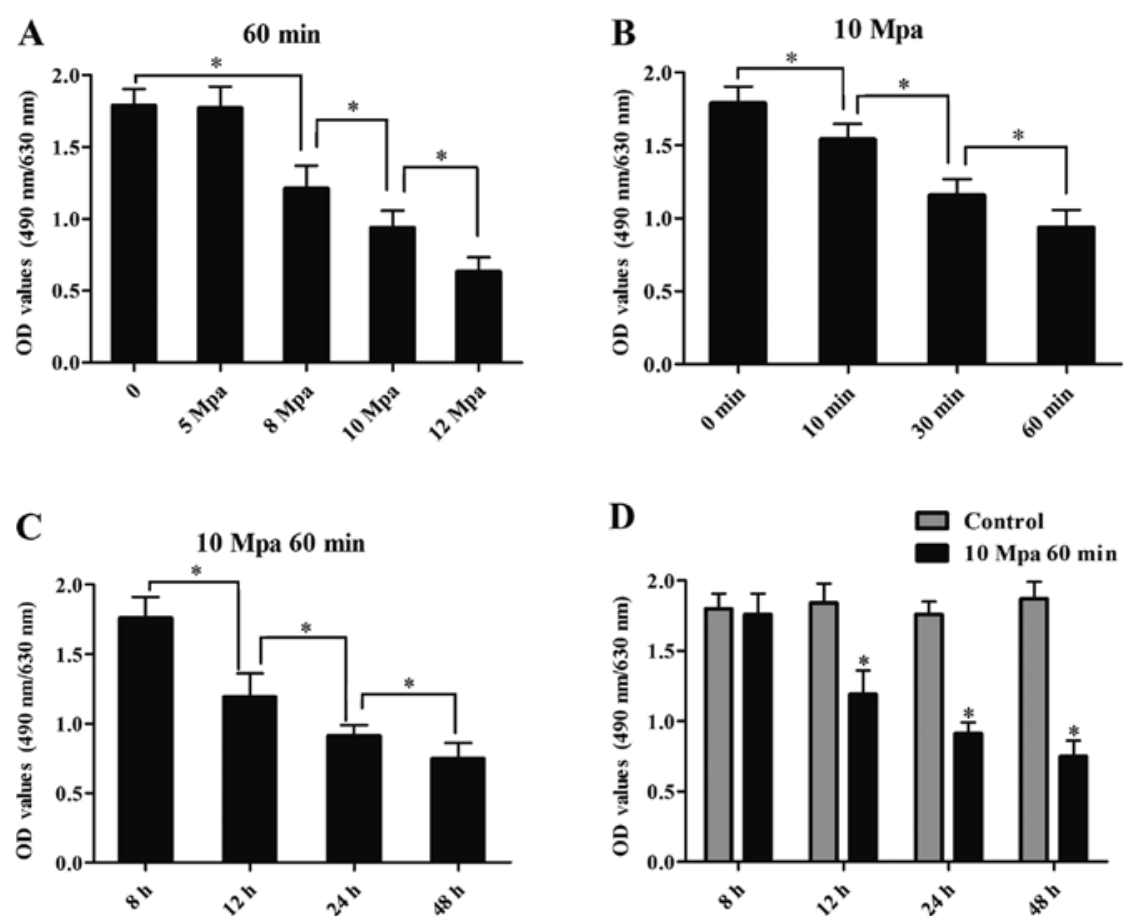

Figure 2. Effects of mechanical injury on the viability of human chondrocytes. (A) The OD values of normal chondrocytes exposed to 5, 8, 10 and $12 \mathrm{Mpa}$ of mechanical pressure for $60 \mathrm{~min}$, at $24 \mathrm{~h}$ following exposure to mechanical pressurey ( $\mathrm{P}<0.05$; bars, means $\pm \mathrm{SD} ; \mathrm{n}=3$ ). (B) The OD values of normal chondrocytes exposed to $10 \mathrm{Mpa}$ of mechanical pressure for 10,30 or $60 \mathrm{~min}$, at $24 \mathrm{~h}$ following exposure to mechanical pressure ("P<0.05; bars, means $\pm \mathrm{SD}$; $\mathrm{n}=3$ ). (C) The OD values of normal chondrocytes exposed to $10 \mathrm{Mpa}$ mechanical pressure for $60 \mathrm{~min}$, at $8,12,24$ and $48 \mathrm{~h}$ following exposure to mechanical pressure ("P<0.05; bars, means $\pm \mathrm{SD} ; \mathrm{n}=3$ ). (D) The OD values of the normal chondrocytes exposed to $10 \mathrm{Mpa}$ mechanical pressure for $60 \mathrm{~min}$, at $8,12,24$ and $48 \mathrm{~h}$ following exposure to mechanical pressure ( $\mathrm{P}<0.05$; bars, means $\pm \mathrm{SD} ; \mathrm{n}=3$ ). Control, non-loaded cells.

cantly decreased in a time-dependent manner after loading of $10 \mathrm{Mpa}$ mechanical pressure $(\mathrm{P}<0.05)$ (Fig. 2B). Based on the preliminary results, the mechanical pressure was set at $10 \mathrm{MPa}$ for $60 \mathrm{~min}$ in the main experiment. The OD values of the chondrocytes loaded with $10 \mathrm{MPa}$ of pressure for $60 \mathrm{~min}$ were reduced in a time-dependent manner $(\mathrm{P}<0.05)$ (Fig. 2C). Compared with the non-loaded chondrocytes, the OD values of the chondrocytes loaded with $10 \mathrm{MPa}$ of pressure for $60 \mathrm{~min}$ were reduced in a time-dependent manner at 12, 24 and $48 \mathrm{~h}$ after pressure injury was sustained $(\mathrm{P}<0.05)$ (Fig. 2D).

Mechanical pressure injury increases the expression levels of miR-146a and VEGF and decreases the levels of Smad4 in human chondrocytes. To identify the miRNAs involved in the process of injury sustained by chondrocytes due to mechanical pressure and their role in the pathogenesis of OA, we screened for the miRNAs which responded to exposure to mechanical pressure (10 Mpa, $60 \mathrm{~min}$ ) in the human chondrocytes. This is a novel cell injury model to mimic mechanical pressure injury substained by chondrocytes related to the progression of OA in vitro. The expression profiles of miRNAs and the transcription profiles in mechanically injured chondrocytes at $48 \mathrm{~h}$ following exposure to mechanical pressure were investigated by miRNA and gene microarray analysis. A series of miRNAs and genes was found to have altered expression levels in response to mechanical pressure injury. The results revealed that there was a significant difference in the expression of miR-146a between the mechanically injured and normal human chondrocytes with a 406 fold change in expression (data not shown); this is in accordance with the results of a previous study which demonstrated that miR-146a mediates inflammatory response (16). Its expression is higher in OA cartilage than in normal cartilage (33). Bioinformatics analysis revealed that $\mathrm{Smad} 4$ is a potential target gene of miR-146a. The gene microarray report indicated that the expression levels of Smad4 were downregulated and the expression levels of VEGF were upregulated (data not shown). Thus, we selected miR-146a for further investigation.

Mechanical pressure injury (10 Mpa, $60 \mathrm{~min}$ ) rapidly induced miR-146a and VEGF expression and inhibited Smad4 expression in human chondrocytes, and their expression gradually increased and decreased, respectively over a 48-h time course following the exposure of the chondrocytes to mechanical pressure (Fig. 3A-C), which was consistent with the microarray results (data not shown). Mechanical pressure injury stimulated the VEGF protein levels and inhibited the Smad4 protein levels (Fig. 3D) in a time-dependent manner.

Upregulation of miR-146a and validation for miR-146a oligonucleotide transfection in human chondrocytes. To investigate the function of miR-146a, we transfected the human chondrocytes with miR-146a mimics, negative control mimics, miR-146a inhibitors or negative control inhibitors. These oligonucleotides could be observed with a fluorescence microscope (Olympus China, Beijing, China) as there was a FAM fluorescent label in their 5' oligonucleotide structure. At $24 \mathrm{~h}$ after transfection, fluorescence microscopy revealed that the transfection efficiency of the miR-146a mimics in the 
A

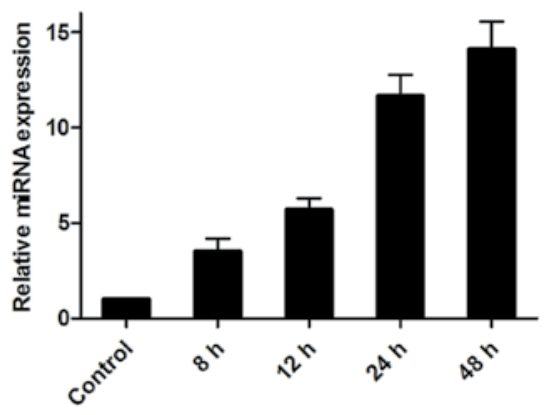

C

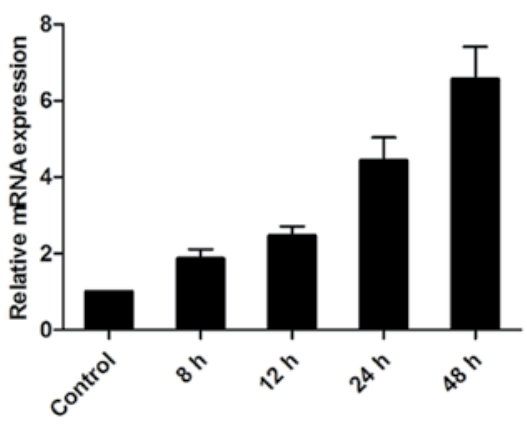

B

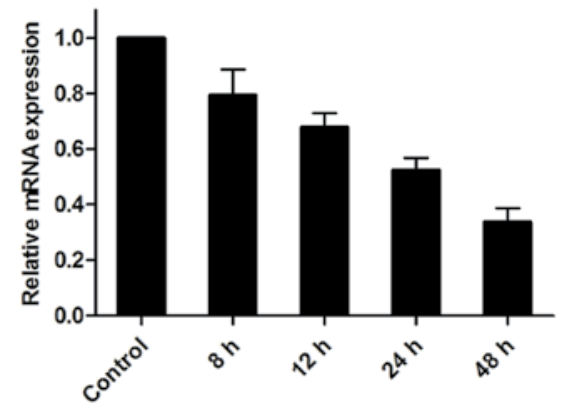

D

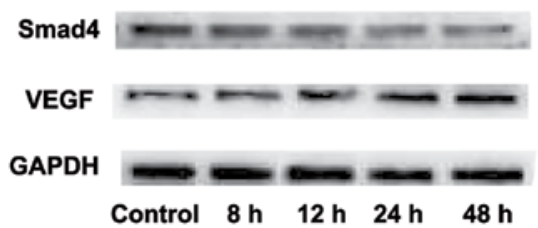

Figure 3. Mechanical pressure injury rapidly induced miR-146a and vascular endothelial growth factor (VEGF) expression and downregulated Smad4 expression in a time-dependent manner after the injury was sustained. Human chondrocytes were loaded with mechanical pressure (10 Mpa, 60 min). Expression levels of miR-146a, Smad4 and VEGF were monitored by RT-qPCR and western blot analysis at the indicated times after the injury was sustained. (A) miR-146a expression was significantly increased over a 48 -h time course $(\mathrm{P}<0.05, \mathrm{n}=3)$. $(\mathrm{B}) \mathrm{mRNA}$ levels of Smad4 were reduced after mechanical pressure injury $(\mathrm{P}<0.05$, $\mathrm{n}=3$ ). (C) VEGF expression was upregulated by mechanical pressure injury $(\mathrm{P}<0.05, \mathrm{n}=3)$. (D) Protein levels of Smad4 and VEGF were, respectively, decreased and increased in chondrocytes in a time-dependent manner after mechanical pressure injury. GAPDH was used as an internal control. Control, non-loaded cells.

human chondrocytes was $>80 \%$ (Fig. 4A), and the transfection efficiency of the other groups was similar (data not shown). miR-146a was significantly knocked down or overexpressed by transfection with miRNA inhibitors or miRNA mimics compared with the negative control roups $(\mathrm{P}<0.01)$ (Fig. $4 \mathrm{~B}$ and C).

miR-146a targets Smad4 through a seed site in the 3'-UTR of Smad4 mRNA. To determine whether miR-146a regulates the expression of Smad4 and VEGF, we transfected the human chondrocytes with miR-146a mimics (146aMi), negative control mimics (MiNC), miR-146a inhibitors (146aIn) or negative control inhibitors (InNC). The results indicated that miR-146a regulates the expression of Smad4 and VEGF in an opposite manner. The overexpression of miR-146a inhibited Smad4 expression and stimulated the VEGF mRNA and protein levels (Fig. 5A-C). By contrast, the knockdown of miR-146a by miR-146a inhibitor increased Smad4 expression and inhibited the VEGF mRNA and protein levels in the human chondrocytes (Fig. 5D-F).

Using miRNA target prediction software as previously described (43), we examined the potential targets of miR-146a by searching the PicTar and miRanda, as well as the TargetScan databases. After consulting the microarray results (data not shown), among the candidate targets, we identified a potential miR-146a binding sequence in the 3'-UTR of Smad4, which contains a putative region that matches the seed sequence of miR-146a (Fig. 6A). Furthermore, to determine whether Smad4 is indeed the target of miR-146a through this seed sequence, we constructed luciferase reporter plasmids harboring the wild-type 3'-UTR and the mutant 3'-UTR (Fig. 6A). While luciferase activity of the mutant $3^{\prime}$-UTR reporter was not statistically significant between the miR-146a mimic and the negative control group (MiNC), the reporter luciferase activity of the wild-type 3'-UTR was significantly inhibited by miR146a in the miR-146a mimic group (146aMi) compared with the negative control group (MiNC) (Fig. 6B). The luciferase activity of the wild-type 3'-UTR reporter was significantly increased in the miR-146a inhibitor (146aIn)-transfected cells compared with the inhibitor negative control group (InNC)transfected cells, and this increase was markedly reduced in the mutant 3'-UTR (Fig. 6C). In summary, Smad4 is a direct target of miR-146a.

Effects of mechanical pressure injury and miR-146a on apoptosis of human chondrocytes. The number of apoptotic cells was counted and compared between the non-loaded normal human chondrocytes and the chondrocytes which were exposed to $10 \mathrm{MPa}$ of mechanical pressure for $60 \mathrm{~min}$ by flow cytometry at $8,12,24$ or 48 h after the injury was sustained. The percentage of apoptotic chondrocytes loaded with $10 \mathrm{MPa}$ of pressure for $60 \mathrm{~min}$ significantly increased in a time-dependent manner $(\mathrm{P}<0.01)$ (Fig. 7A).

Since mechanical pressure injury stimulates apoptosis and the expression levels of miR-146a in chondrocytes, we examined whether the expression of miR-146a affects chondrocyte 

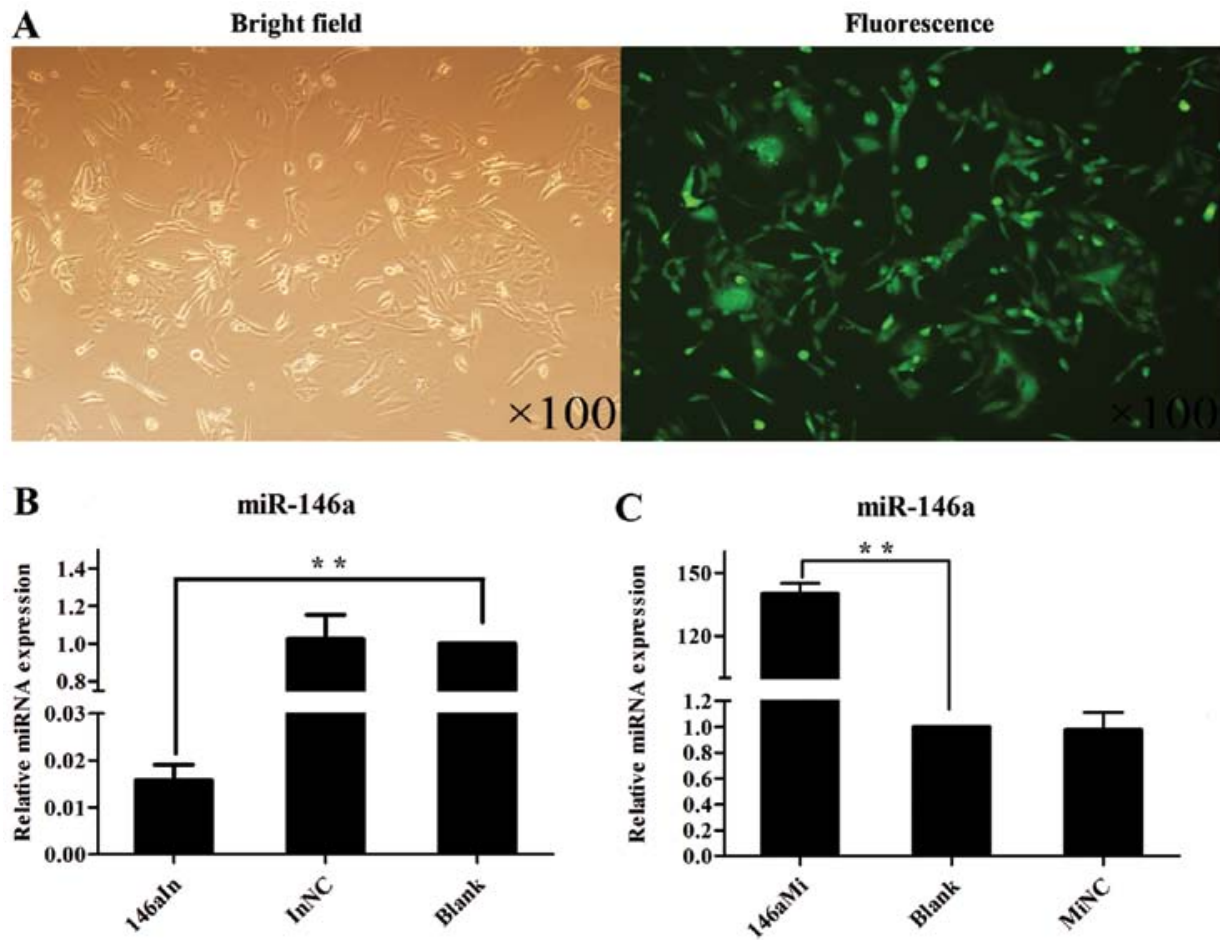

Figure 4. mMiR-146a oligonucleotide transfection and miR-146a expression in human chondrocytes. (B and C) Human chondrocytes were transfected with miR-146a mimics (146aMi), negative control mimics (MiNC), miR-146a inhibitors (146aIn), negative control inhibitors (InNC) and vacuous oligonucleotide (Blank). (A) Human chondrocytes were observed with white bright (left panel) and green fluorescence assay (right panel) in the same vision using a fluorescence microscope (magnification, $\mathrm{x} 100$ ) at $24 \mathrm{~h}$ after transfection. (B) miR-146a expression levels were evaluated by RT-qPCR in the chondrocytes transfected with miR146a inhibitors (146aIn) and negative control inhibitors ( $\mathrm{InNC})\left({ }^{* *} \mathrm{P}<0.01\right.$; bars, means $\left.\pm \mathrm{SD} ; \mathrm{n}=3\right)$. (C) miR-146a expression levels were evaluated by RT-qPCR analysis in chondrocytes transfected with miR-146a mimics (146aMi) and negative control mimics (MiNC) (** $\mathrm{P}<0.01$; bars, means $\pm \mathrm{SD} ; \mathrm{n}=3)$.
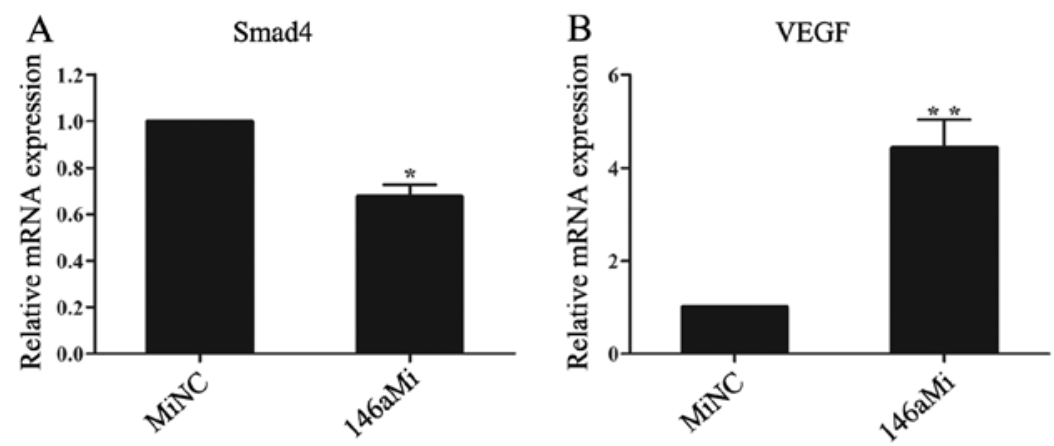

C
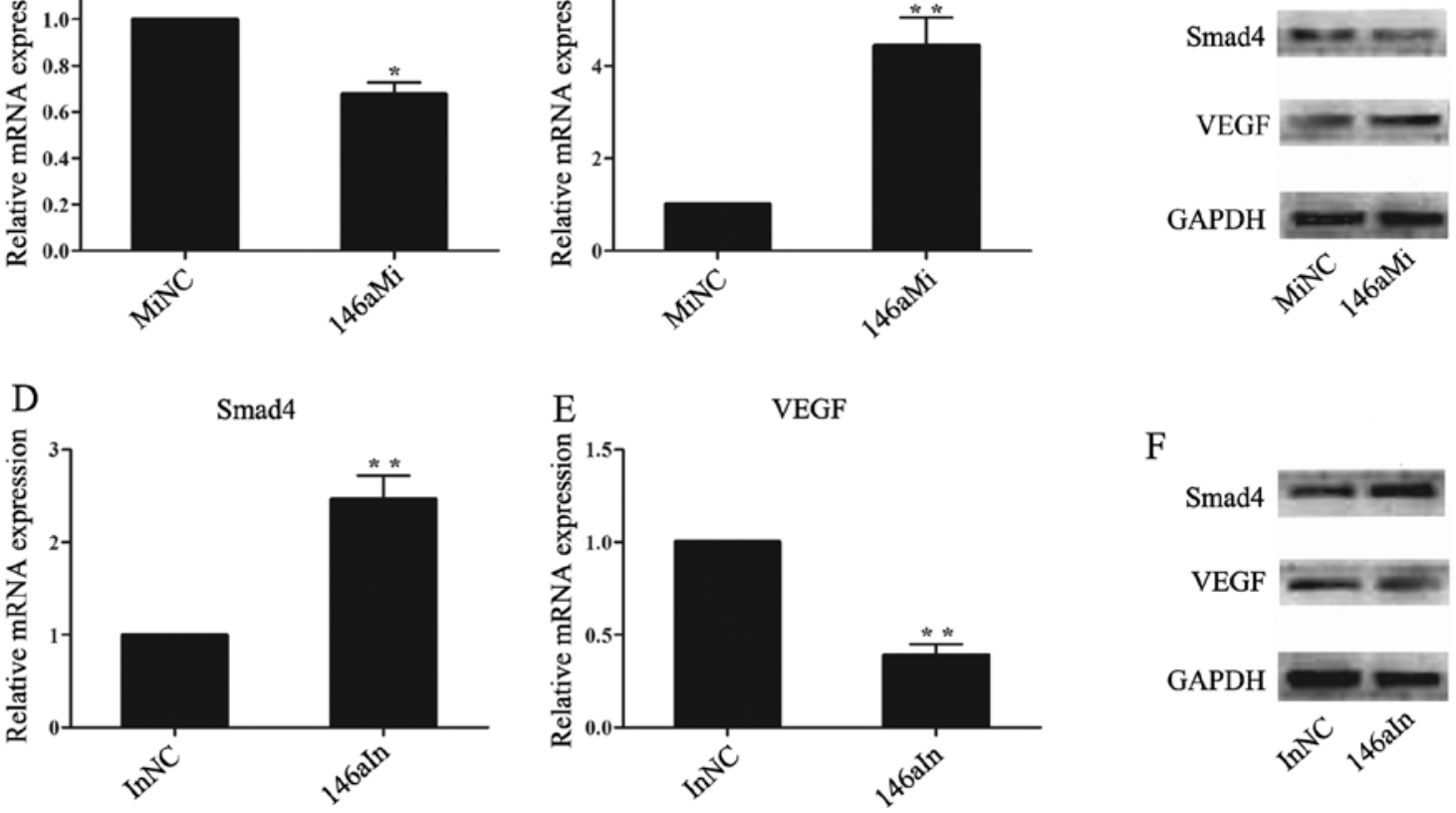

Figure 5. Expression levels of miR-146a, Smad4 and VEGF were monitored by RT-qPCR and western blot analysis at $24 \mathrm{~h}$ after transfection. (A-C) Human chondrocytes were transfected with miR-146a mimics (146aMi) and negative control mimics (MiNC). (D-F) Human chondrocytes were transfected with miR-146a inhibitors (146aIn) and negative control inhibitors (InNC). (A) Overexpression of miR-146a significantly inhibited Smad4 mRNA levels ("P<0.05; bars, means $\pm \mathrm{SD} ; \mathrm{n}=3$ ). (B) VEGF mRNA expression was upregulated by the overexpression of miR-146a ( ${ }^{* *} \mathrm{P}<0.01$; bars, means $\left.\pm \mathrm{SD} ; \mathrm{n}=3\right)$. (C) Protein levels of Smad4 and VEGF were, respectively, decreased and increased in chondrocytes transfected with miR-146a mimics. GAPDH was used as an internal control. (D) mRNA levels of Smad4 were increased by the knockdown of miR-146a with miR-146a inhibitor ${ }^{* *} \mathrm{P}<0.01$; bars, means $\pm \mathrm{SD}$; $n=3$ ). (E) mRNA levels of VEGF were reduced by the knockdown of miR-146a with miR-146a inhibitor $\left({ }^{* * *} \mathrm{P}<0.01\right.$; bars, means $\pm \mathrm{SD} ; \mathrm{n}=3$ ). (F) Protein levels of Smad4 and VEGF were respectively increased and decreased in chondrocytes transfected with miR-146a inhibitor. GAPDH was used as an internal control. 

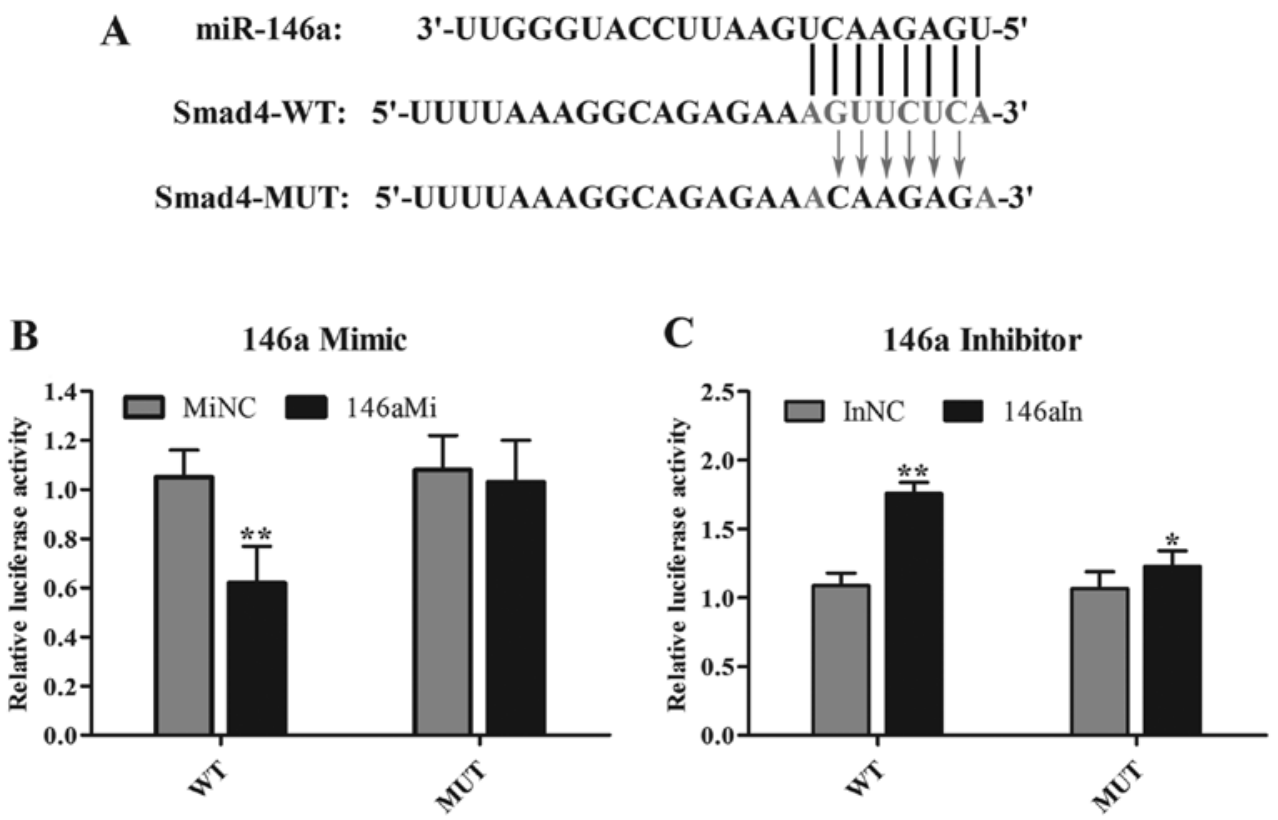

Figure 6. Smad4 is a direct target of miR-146a. (A) Schematic representation of the sequence alignment of the wild-type Smad4 3'-UTR (light color) indicating the binding site of the miR-146a and the mutant Smad4 3'-UTR for pMIR-report (WT, wild-type; MUT, mutant). (B) Compared with negative control group (MiNC), miR-146a mimics (146aMi) significantly inhibited the reporter luciferase activity of the wild-type Smad4 3'-UTR ("* $\mathrm{P}<0.01$; bars, means $\pm \mathrm{SD}$; $\mathrm{n}=3$ ) but not that of the mutant Smad4 3'-UTR (P>0.05; bars, means $\pm \mathrm{SD} ; \mathrm{n}=3$ ). (C) Compared with inhibitor negative control group (InNC), the reporter luciferase activity of the wild-type Smad4 3'-UTR was significantly increased by miR-146a inhibitor (146aIn) ( ${ }^{* * *} \mathrm{P}<0.01$; bars, means $\pm \mathrm{SD}$; $\mathrm{n}=3$ ) than that of the mutant Smad4 3'-UTR $\left({ }^{*} \mathrm{P}<0.05 ;\right.$ bars, means $\left.\pm \mathrm{SD} ; \mathrm{n}=3\right)$.
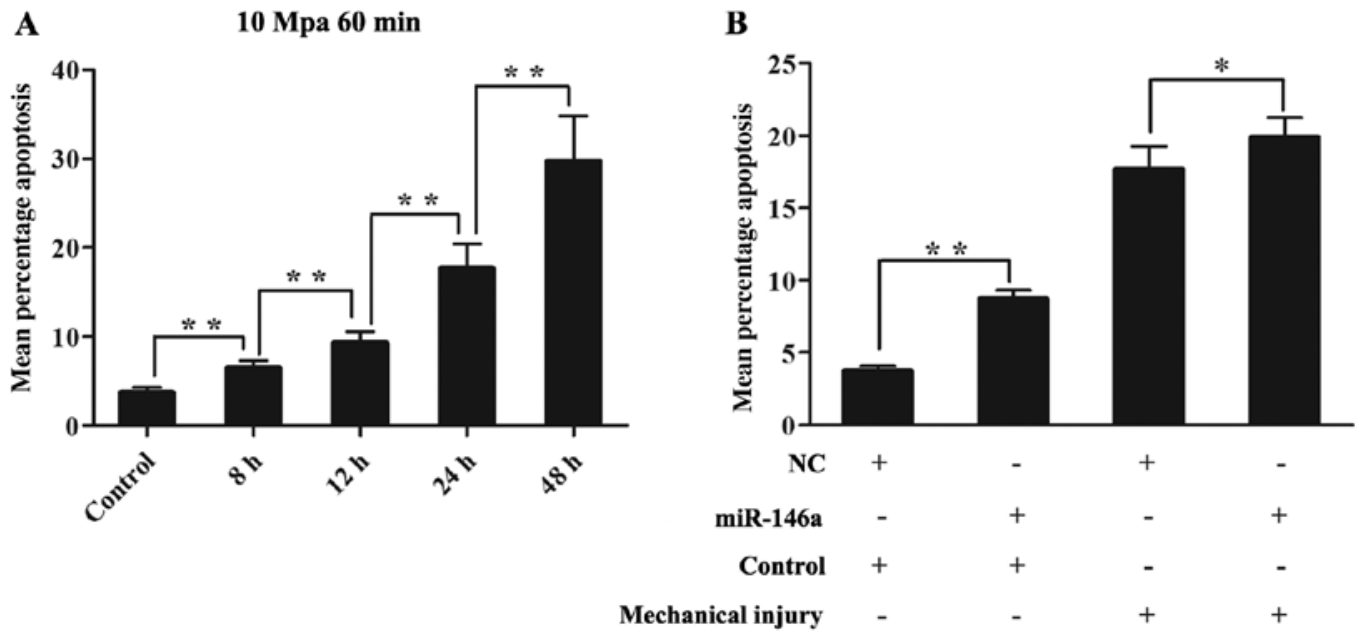

Figure 7. Mechanical pressure injury and miR-146a lead to apoptosis of human chondrocytes. (A) Compared with the control cells, the percentage of apoptotic chondrocytes loaded with $10 \mathrm{MPa}$ of pressure for $60 \mathrm{~min}$ significantly increased in a time-dependent manner after injury was sustained (** $\mathrm{P}<0.01 ;$ bars, means $\pm \mathrm{SD} ; \mathrm{n}=3$ ). (B) Human chondrocytes were loaded with mechanical pressure (10 Mpa, $60 \mathrm{~min})$, at $12 \mathrm{~h}$ after transfection with miR-146a mimics or negative control. The number of apoptotic cells was counted by flow cytometry, at $12 \mathrm{~h}$ after the injury was sustained or at $24 \mathrm{~h}$ after transfection. Compared with the control cells, the percentage of apoptotic chondrocytes overexpressing miR-146a significantly increased $24 \mathrm{~h}$ after transfection with miR-146a mimics ${ }^{* *} \mathrm{P}<0.01$; bars, means $\pm \mathrm{SD} ; \mathrm{n}=3$ ). Compared with the chondrocytes exposed to mechanical pressure and transfected with the negative control, the percentage of apoptotic chondrocytes overexpressing miR-146a significantly increased $24 \mathrm{~h}$ after transfection ( $\mathrm{P}<0.05$; bars, means $\pm \mathrm{SD} ; \mathrm{n}=3)$.

apoptosis. The overexpression of miR-146a in the chondrocytes induced a significant increase in the percentage of apoptotic chondrocytes at $24 \mathrm{~h}$ after transfection $(\mathrm{P}<0.01)$ (Fig.7B), and markedly increased the percentage of apoptotic chondrocytes exposed to mechanical pressure (10 Mpa, $60 \mathrm{~min}$, injured at $12 \mathrm{~h}$ after transfection) at $12 \mathrm{~h}$ after the injury was sustained $(\mathrm{P}<0.05)$ (Fig. 7B). These results indicate that miR-146a plays a role in mediating mechanical injury-induced apoptosis in human chondrocytes.

Mechanical pressure injury regulates Smad4 and VEGF expression and chondrocyte apoptosis through miR-146a in human chondrocytes. To demonstrate the role of miR-146a in mediating mechanical injury sustained by chondrocytes, we 
A

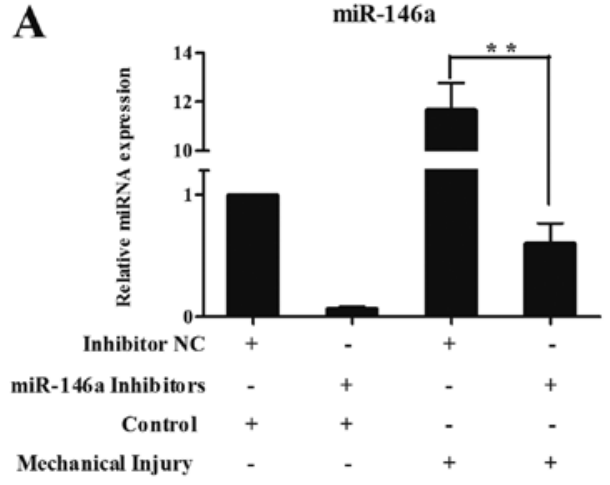

C

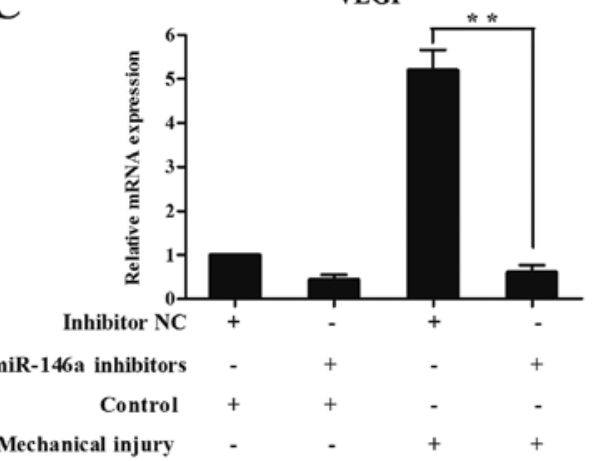

B

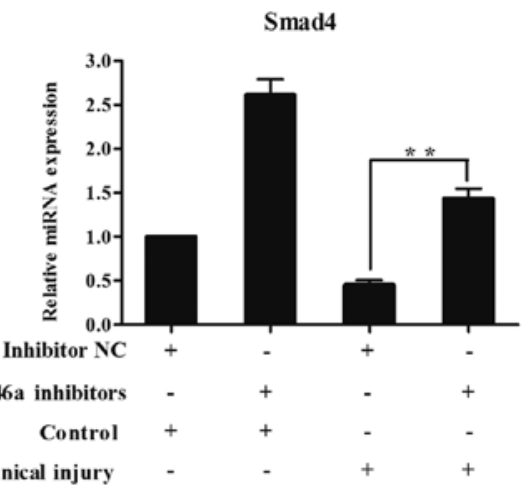

D

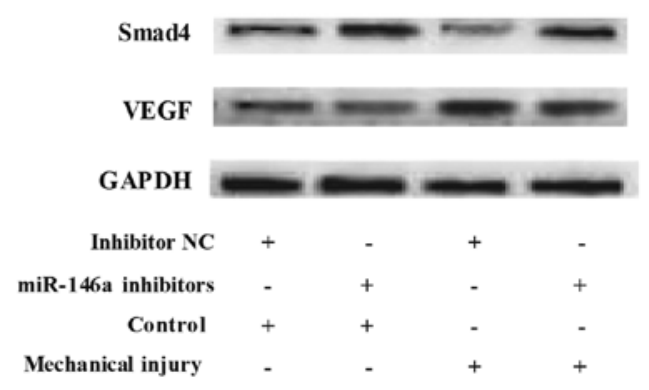

Figure 8. The inhibition of miR-146a attenuates the downregulation of Smad4, the upregulation of VEGF, and the increase in chondrocyte apoptosis after mechanical injury. Chondrocytes were transfected with miR-146a inhibitors and loaded with or without mechanical pressure (10 Mpa, 60 min). (A) Compared with mechanically injured chondrocytes transfected with inhibitor negative control, miR-146a inhibitors decreased the upregulation of miR-146a after mechanical injury ( ${ }^{* *} \mathrm{P}<0.01$; bars, means $\pm \mathrm{SD} ; \mathrm{n}=3$ ). (B) Compared with mechanically injured chondrocytes transfected with inhibitor negative control, the downregulation of Smad4 mRNA expression was markedly increased by miR-146a inhibitors after mechanical injury ${ }^{* *} \mathrm{P}<0.01$; bars, means $\pm \mathrm{SD}$; $\mathrm{n}=3$ ). (C) Compared with mechanically injured chondrocytes transfected with inhibitor negative control, VEGF expression was markerdly reduced by miR-146a inhibitors after mechanical injury ( ${ }^{* *} \mathrm{P}<0.01$; bars, means $\pm \mathrm{SD} ; \mathrm{n}=3$ ). (D) Compared with mechanically injured chondrocytes transfected with inhibitor negative control, miR-146a inhibitors counteracted the downregulation of Smad4 and upregulation of VEGF at the protein level after mechanical injury.

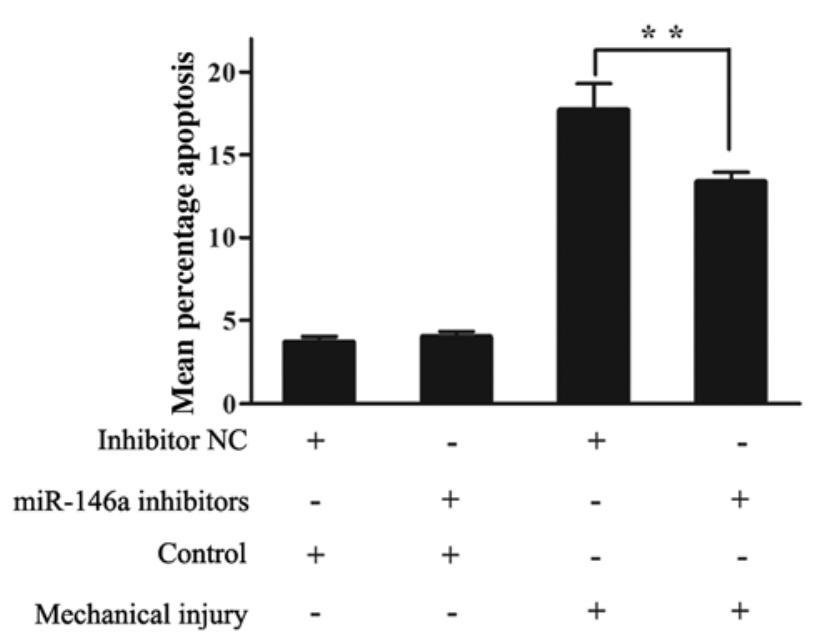

Figure 9. Compared with mechanically injured chondrocytes transfected with inhibitor negative control, the promoter effect of chondrocyte apoptosis was significantly counteracted by miR-146a inhibitors after mechanical injury $\left({ }^{* *} \mathrm{P}<0.01\right.$; bars, means $\left.\pm \mathrm{SD} ; \mathrm{n}=3\right)$.

used miR-146a inhibitor to block its expression in human chondrocytes. Human chondrocytes were loaded with mechanical pressure (10 Mpa, $60 \mathrm{~min}$ ) $12 \mathrm{~h}$ following transfection with
miR-146a inhibitor. The expression levels of miR-146a, Smad4 and VEGF were monitored by RT-qPCR and western blot analysis at $12 \mathrm{~h}$ after the injury was sustained. The knockdown of miR-146a with the inhibitor significantly suppressed the upregulation of miR-146a expression induced by mechanical injury (Fig. 8A). Transfection with the miR-146a inhibitor significantly increased Smad4 mRNA expression, while the Smad4 mRNA levels were inhibited following mechanical injury (Fig. 8B). While mechanical injury markerly increased the VEGF mRNA levels, transfection with miR-146a inhibitor reversed this effect (Fig. 8C). The knockdown of endogenous miR-146a induced similar effects on Smad4 and VEGF protein levels as on their mRNA levels following mechanical injury (Fig. 8D). Compared with the mechanically injured chondrocytes transfected with the negative control inhibitor, the percentage of apoptotic chondrocytes transfected with the miR-146a inhibitor decreased $12 \mathrm{~h}$ after the injury was sustained (Fig. 9). These results indicte that miR-146a is involved in human chondrocyte apoptosis in response to mechanical injury by regulating Smad4 and VEGF expression.

Mechanical pressure injury upregulates VEGF expression and chondrocyte apoptosis through Smad4 in human chondrocytes. To determine whether miR-146a mediates the upregulation of VEGF and chondrocyte apoptosis through 


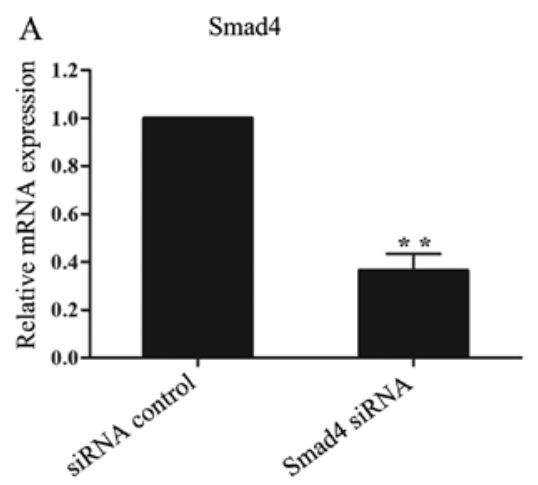

B
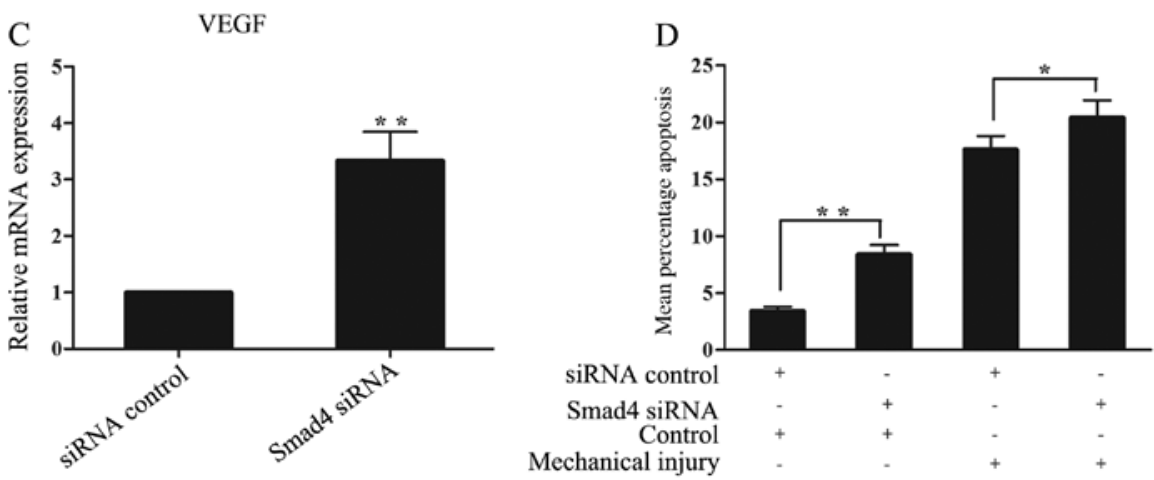

Figure 10. VEGF expression and chondrocyte apoptosis is regulated by Smad4. Knockdown of Smad4 by RNAi increased VEGF expression at the protein level. Chondrocytes were transfected with siRNA against Smad4 or control siRNA (Ctrl). (A) Smad4 siRNA effectively reduced the expression of Smad4 mRNA. (B) Inhibition of Smad4 increased the protein level of VEGF. GAPDH was used as an internal control. (C) mRNA levels of VEGF were reduced by the knockdown of Smad4 with Smad4 siRNA ( ${ }^{* *} \mathrm{P}<0.01$; bars, means $\left.\pm \mathrm{SD} ; \mathrm{n}=3\right)$. (D) Inhibition of Smad4 increased the percentage of apoptotic cells in non-loaded chondrocytes $($ ** $<<0.01$; bars, means $\pm \mathrm{SD} ; \mathrm{n}=3)$; Compared with the mechanically injured chondrocytes transfected with control siRNA, the knockdown of Smad4 effectively increased the percentage of apoptotic cells ( $\mathrm{P}<0.05$; means $\pm \mathrm{SD} ; \mathrm{n}=3)$.

Smad4 following mechanical pressure injury, the chondrocytes were transfected with Smad4 siRNA to inhibit Smad4 expression. Transfection with Smad4 siRNA reduced the levels of both Smad4 mRNA (Fig. 10A) and protein (Fig. 10B). The knockdown of Smad4 significantly increased the VEGF mRNA and protein levels (Fig. 10B and C), and induced a marked increase in the percentage of apoptotic cells in the non-loaded chondrocytes, at $24 \mathrm{~h}$ after transfection $(\mathrm{P}<0.01)$ (Fig. 10D). Compared with the mechanically injured chondrocytes transfected with control siRNA, the inhibition of Smad4 markedly increased the percentage of apoptotic cells (10 Mpa, $60 \mathrm{~min}$, injured at $12 \mathrm{~h}$ after transfection) at $12 \mathrm{~h}$ after the injury was sustained $(\mathrm{P}<0.05)$ (Fig. 10D). These results indicate that Smad4 mediates the upregulation of VEGF and chondrocyte apoptosis.

\section{Discussion}

Blunt mechanical force injury sustained by the articular cartilage, often results from accidents or sports injuries (44). Mechanical force injury sustained by chondrocytes is associated with local inflammatory reactions and represents a major risk factor for the development of OA (45). In the present study, we developed a novel chondrocyte model of biomechanically defined mechanical pressure-induced human mechanical injury for in vitro studies, and demonstrate for the first time that miR-146a is upregulated by experimentally-induced chondrocyte mechanical injury in this model. To the best of our knowledge, this is the first time that miR-146a is identified as a mechano-responsive miRNA in chondrocytes. However, it remains to be determined whether miR-146a is responsive to the overload of mechanical stimuli in addition to the gene expression of catabolic and anabolic markers and the release of proinflammatory mediators.

In response to overloading external stimuli, cells are forced to die in different ways $(46,47)$. In animal and human cartilage injury models, mechanical stimuli represent important regulators of chondrocyte function and induce mediators of inflammation and chondrocyte death (48-51). In the pathological process of chondrocyte mechanical injury, parts of damaged chondrocytes undergo necrosis in the early postinjury phase. Parts of the remaining demaged chondrocytes are prone to apoptosis $(12,52)$. A number of studies have shown that the rate of chondrocyte apoptosis is increased in OA cartilage and have authenticated the role of apoptosis in the pathogenesis of OA $(9,11,52-55)$. In the present study, chondrocyte viability significantly decreased and the percentage of apoptotic chondrocytes increased, separately, in a time-dependent manner following the exposure of chondrocytes to mechanical pressure.

However, the process of programmed cell death occurs through the activation of the caspase cascade, and may be blocked if one of the proteins involved in executing apoptosis is genetically impaired or chemically inhibited, or if the apoptotic machinery is not properly operated under specific 
conditions, such as ischemia and microbial infection, which suggests possible targets for novel therapeutic strategies (56). Thus, modulation of the mechanisms mediated by substances inducing apoptosis is being considered as a novel strategy for the treatment of OA. Our study focused on miR-146a, Smad4 and VEGF following the screening of differentially expressed genes and miRNAs. Mechanical pressure injury increased the expression levels of miR-146a and VEGF and decreased the levels of Smad4 in the chondrocytes in a time-dependent manner. The results revealed that the expression levels of Smad4 were inversely related to the miR-146a and VEGF levels. Our follow-up experiments suggested that Smad4 is a direct target of miR-146a for post-transcriptional regulation. Furthermore, our data suggest that miR-146a regulates chondrocyte apoptosis by inhibiting Smad4. Yet, the mechanisms through which $\mathrm{Smad} 4$ reduces chondrocyte apoptosis remain unknown. It has been shown previously that Smad4 is a key mediator in transmitting signals from TGF- $\beta$ (57). Furthermore, extracellular signal-regulated protein kinases 1 and $2($ ERK1/2) are cental members of the mitogen-activated protein kinase superfamily that can mediate cell proliferation and apoptosis $(58,59)$. Moreover, previous studies have shown that the TGF- $\beta$ stimulation of ERK $1 / 2$ phosphorylation is independent of Smad4 (60). The knockdown of Smad4 by miR-146a may thus block the activation of ERK1/2 to regulate chondrocyte apoptosis.

Mechanical signals are important for normal cartilage to maintain tissue integrity and homeostasis $(61,62)$. Chondrocytes respond to changes in the levels of proinflammatory mediators and mechanical signals in OA $(63,64)$. Pro-inflammatory mediators inhibit homeostatic mechanisms and supress cartilage repair and chondrocyte viability. However, the physiological levels of mechanical forces induce matrix synthesis and chondrocyte proliferation (65). Previous studies have suggested that VEGF, an important synovial and cartilage vascularization factor (66), appears to also be involved in the process of OA (67), and can be induced by mechanical forces to ERK1/2 activation for sustaining the effects of mechanical signals for mechanisms underlying reparative actions. In our further observation, the data indicated that the upregulation of VEGF induced by miR-146a overexpression is mediated by Smad4 in mechanically injured chondrocytes. These reuslts are consistent with those of previous studies, showing that Smad4 inhibits VEGF expression and suppresses tumorigenicity through the inhibition of angiogenesis in human pancreatic and gastrointestinal carcinoma cells (68-70). Of note, while the miR-146a inhibitor or Smad4 siRNA markerly affected the mechanical injury regulation of VEGF, the inhibition of miR-146a or Smad4 did not completely counteract the induction of VEGF in response to the overloading mechanical. This suggests that, in addition to miR-146a and Smad4, other factors are involved in mediating the mechanical injury regulation of VEGF and Smad4. Evidence suggests that chondrocytic mechanosensing is competent of recognizing and esponding to signals of various intensities to differentially mediate cartilage repair and pathologies (71). Furthermore, mechanical injury stimuli includes plural mechanical signals. Moreover, singleness mechanical signal-stimulates activation of cells is a complex rapid process and leads to the activation of multiple intracel- lular signaling cascades, flow channels and genes (72-74). We speculate that the induction of VEGF by mechanical injury may partially depend on an unknown activation of cells by some mechanical signal.

The results of this study demonstrate that miR-146a is overexpressed in an experimental chondrocyte model of human mechanical injury, accompanied by the upregulation of VEGF and the downregulation of Smad4 in vitro. miR-146a is involved in human chondrocyte apoptosis in response to mechanical injury, and may contribute to the mechanical injury sustained by chondrocytes and the pathogenesis of OA by increasing the levels of VEGF and damaging the TGF- $\beta$ signaling pathway through the targeted inhibition of Smad4 in human chondrocytes. These data may provide a novel signaling cascade that links miR-146a-mediated mechanical injury stimuli to Smad4-dependent cell apoptosis in human chondrocytes through a mechanism involving TGF- $\beta$, ERK1/2 and VEGF, and raise the possibility that miR-146a may be a therapeutic target for the treatment of OA.

\section{Acknowledgements}

The authors wish to thank Yunyan Liu, Yanhua Wen and Qiong Ma for providing excellent technical assistance.

\section{References}

1. Ashford S and Williard J: Osteoarthritis: A review. Nurse Pract 39: 1-8, 2014

2. Anderson DD, Chubinskaya S, Guilak F, et al: Post-traumatic osteoarthritis: improved understanding and opportunities for early intervention. J Orthop Res 29: 802-809, 2011.

3. Martin JA and Buckwalter JA: Post-traumatic osteoarthritis: the role of stress induced chondrocyte damage. Biorheology 43: 517-521, 2006.

4. Seol D, McCabe DJ, Choe H, et al: Chondrogenic progenitor cells respond to cartilage injury. Arthritis Rheum 64: 3626-3637, 2012.

5. Conde J, Scotece M, Gomez R, Lopez V, Gomez-Reino JJ and Gualillo O: Adipokines and osteoarthritis: novel molecules involved in the pathogenesis and progression of disease. Arthritis 2011: 203901, 2011.

6. Hogrefe C, Joos H, Maheswaran V, Durselen L, Ignatius A and Brenner RE: Single impact cartilage trauma and TNF-alpha: interactive effects do not increase early cell death and indicate the need for bi-/multidirectional therapeutic approaches. Int J Mol Med 30: 1225-1232, 2012.

7. Joos H, Hogrefe C, Rieger L, Durselen L, Ignatius A and Brenner RE: Single impact trauma in human early-stage osteoarthritic cartilage: implication of prostaglandin $\mathrm{D} 2$ but no additive effect of IL-1 $\beta$ on cell survival. Int J Mol Med 28: 271-277, 2011.

8. Leucht F, Durselen L, Hogrefe C, et al: Development of a new biomechanically defined single impact rabbit cartilage trauma model for in vivo-studies. J Invest Surg 25: 235-241, 2012.

9. Heraud F, Heraud A and Harmand MF: Apoptosis in normal and osteoarthritic human articular cartilage. Ann Rheum Dis 59: 959-965, 2000

10. Colwell CW Jr, D'Lima DD, Hoenecke HR, et al: In vivo changes after mechanical injury. Clin Orthop Relat Res (391 Suppl): S116-S123, 2001.

11. D'Lima DD, Hashimoto S, Chen PC, Colwell CW Jr and Lotz MK: Human chondrocyte apoptosis in response to mechanical injury. Osteoarthritis Cartilage 9: 712-719, 2001.

12. Tew SR, Kwan AP, Hann A, Thomson BM and Archer CW: The reactions of articular cartilage to experimental wounding: role of apoptosis. Arthritis Rheum 43: 215-225, 2000.

13. D'Lima DD, Hashimoto S, Chen PC, Lotz MK and Colwell CW Jr: Cartilage injury induces chondrocyte apoptosis. J Bone Joint Surg Am 83-A (Suppl 2): 19-21, 2001. 
14. Saito Y, Saito H, Liang G and Friedman JM: Epigenetic alterations and microRNA misexpression in cancer and autoimmune diseases: a critical review. Clin Rev Allergy Immunol: Dec 21, 2013 (Epub ahead of print).

15. Bartel DP: MicroRNAs: target recognition and regulatory functions. Cell 136: 215-233, 2009.

16. Taganov KD, Boldin MP, Chang KJ and Baltimore D NF-kappaB-dependent induction of microRNA miR-146, an inhibitor targeted to signaling proteins of innate immune responses. Proc Natl Acad Sci USA 103: 12481-12486, 2006.

17. Taganov KD, Boldin MP and Baltimore D: MicroRNAs and immunity: tiny players in a big field. Immunity 26: 133-137, 2007.

18. Tsai CY, Allie SR, Zhang W and Usherwood EJ: MicroRNA miR-155 affects antiviral effector and effector Memory CD8 T cell differentiation. J Virol 87: 2348-2351, 2013.

19. Chiyomaru T, Enokida H, Tatarano S, et al: miR-145 and miR-133a function as tumour suppressors and directly regulate FSCN1 expression in bladder cancer. Br J Cancer 102: 883-891, 2010.

20. Murphy AJ, Guyre PM and Pioli PA: Estradiol suppresses NF-kappa B activation through coordinated regulation of let-7a and miR-125b in primary human macrophages. J Immunol 184 5029-5037, 2010.

21. Tili E, Michaille JJ, Cimino A, et al: Modulation of miR-155 and miR-125b levels following lipopolysaccharide/TNF-alpha stimulation and their possible roles in regulating the response to endotoxin shock. J Immunol 179: 5082-5089, 2007.

22. Williams AE, Perry MM, Moschos SA, Larner-Svensson HM and Lindsay MA: Role of miRNA-146a in the regulation of the innate immune response and cancer. Biochem Soc Trans 36 1211-1215, 2008.

23. Miyaki $\mathrm{S}$ and Asahara $\mathrm{H}$ : Macro view of microRNA function in osteoarthritis. Nat Rev Rheumatol 8: 543-552, 2012.

24. Ceribelli A, Nahid MA, Satoh M and Chan EK: MicroRNAs in rheumatoid arthritis. FEBS Lett 585: 3667-3674, 2011

25. Ammari M, Jorgensen C and Apparailly F: Impact of microRNAs on the understanding and treatment of rheumatoid arthritis. Curr Opin Rheumatol 25: 225-233, 2013.

26. Goldring MB and Marcu KB: Epigenomic and microRNA-mediated regulation in cartilage development, homeostasis, and osteoarthritis. Trends Mol Med 18: 109-118, 2012.

27. Okuhara A, Nakasa T, Shibuya H, et al: Changes in microRNA expression in peripheral mononuclear cells according to the progression of osteoarthritis. Mod Rheumatol 22: 446-457, 2012

28. Yu C, Chen WP and Wang XH: MicroRNA in osteoarthritis. J Int Med Res 39: 1-9, 2011.

29. Jones SW, Watkins G, Le Good N, et al: The identification of differentially expressed microRNA in osteoarthritic tissue that modulate the production of TNF-alpha and MMP13. Osteoarthritis Cartilage 17: 464-472, 2009.

30. Iliopoulos D, Malizos KN, Oikonomou P and Tsezou A: Integrative microRNA and proteomic approaches identify novel osteoarthritis genes and their collaborative metabolic and inflammatory networks. PLoS One 3: e3740, 2008.

31. Diaz-Prado S, Cicione C, Muinos-Lopez E, et al: Characterization of microRNA expression profiles in normal and osteoarthritic human chondrocytes. BMC Musculoskelet Disord 13: 144, 2012.

32. Wang JH, Shih KS, Wu YW, Wang AW and Yang CR: Histone deacetylase inhibitors increase microRNA-146a expression and enhance negative regulation of interleukin-lbeta signaling in osteoarthritis fibroblast-like synoviocytes. Osteoarthritis Cartilage 21: 1987-1996, 2013

33. Yamasaki K, Nakasa T, Miyaki S, et al: Expression of microRNA-146a in osteoarthritis cartilage. Arthritis Rheum 60 1035-1041, 2009

34. Nakasa T, Miyaki S, Okubo A, et al: Expression of microRNA146 in rheumatoid arthritis synovial tissue. Arthritis Rheum 58: 1284-1292, 2008.

35. Bhaumik D, Scott GK, Schokrpur S, Patil CK, Campisi J and Benz CC: Expression of microRNA-146 suppresses NF-kappaB activity with reduction of metastatic potential in breast cancer cells. Oncogene 27: 5643-5647, 2008

36. Baoan Ma and Lei Jin: Multifunctional constant-temperature high pressure hydrostatic pressure loading device in in-vitro cel culture. China, utility model patent No. CN 203229539 U. Filed May 16, 2013; issued October 9, 2013.

37. Hashimoto S, Nishiyama T, Hayashi S, et al: Role of p53 in human chondrocyte apoptosis in response to shear strain. Arthritis Rheum 60: 2340-2349, 2009.
38. Moon MH, Jeong JK, Lee YJ, Seol JW and Park SY: Sphingosine-1-phosphate inhibits interleukin-1 $\beta$-induced inflammation in human articular chondrocytes. Int J Mol Med 30: $1451-1458,2012$

39. Takebe K, Nishiyama T, Hayashi $\mathrm{S}$, et al: Regulation of $\mathrm{p} 38$ MAPK phosphorylation inhibits chondrocyte apoptosis in response to heat stress or mechanical stress. Int J Mol Med 27 329-335, 2011.

40. Storch A, Burkhardt K, Ludolph AC and Schwarz J: Protective effects of riluzole on dopamine neurons: involvement of oxidative stress and cellular energy metabolism. J Neurochem 75: 2259-2269, 2000.

41. Griffiths-Jones S, Saini HK, van Dongen S and Enright AJ: miRBase: tools for microRNA genomics. Nucleic Acids Res 36: D154-D158, 2008

42. Bottoni A, Zatelli MC, Ferracin M, et al: Identification of differentially expressed microRNAs by microarray: a possible role for microRNA genes in pituitary adenomas. J Cell Physiol 210: 370-377, 2007.

43. Lewis BP, Burge CB and Bartel DP: Conserved seed pairing, often flanked by adenosines, indicates that thousands of human genes are microRNA targets. Cell 120: 15-20, 2005.

44. Weatherall JM, Mroczek K, McLaurin T, Ding B and Tejwani N: Post-traumatic ankle arthritis. Bull Hosp Jt Dis (2013) 71: 104-112, 2013.

45. Lee JH, Fitzgerald JB, Dimicco MA and Grodzinsky AJ: Mechanical injury of cartilage explants causes specific timedependent changes in chondrocyte gene expression. Arthritis Rheum 52: 2386-2395, 2005

46. Douville NJ, Zamankhan P, Tung YC, et al: Combination of fluid and solid mechanical stresses contribute to cell death and detachment in a microfluidic alveolar model. Lab Chip 11: 609-619, 2011

47. Levin A, Burton-Wurster N, Chen CT and Lust G: Intercellular signaling as a cause of cell death in cyclically impacted cartilage explants. Osteoarthritis Cartilage 9: 702-711, 2001.

48. Honda K, Ohno S, Tanimoto K, et al: The effects of high magnitude cyclic tensile load on cartilage matrix metabolism in cultured chondrocytes. Eur J Cell Biol 79: 601-609, 2000

49. Fermor B, Weinberg JB, Pisetsky DS, Misukonis MA, Banes AJ and Guilak F: The effects of static and intermittent compression on nitric oxide production in articular cartilage explants. J Orthop Res 19: 729-737, 2001.

50. Millward-Sadler SJ, Wright MO, Davies LW, Nuki G and Salter DM: Mechanotransduction via integrins and interleukin-4 results in altered aggrecan and matrix metalloproteinase 3 gene expression in normal, but not osteoarthritic, human articular chondrocytes. Arthritis Rheum 43: 2091-2099, 2000.

51. D'Lima DD, Hashimoto S, Chen PC, Colwell CW Jr and Lotz MK: Impact of mechanical trauma on matrix and cells. Clin Orthop Relat Res (391 Suppl): S90-S99, 2001.

52. Wenger R, Hans MG, Welter JF, Solchaga LA, Sheu YR and Malemud CJ: Hydrostatic pressure increases apoptosis in cartilage-constructs produced from human osteoarthritic chondrocytes. Front Biosci 11: 1690-1695, 2006

53. Islam N, Haqqi TM, Jepsen KJ, et al: Hydrostatic pressure induces apoptosis in human chondrocytes from osteoarthritic cartilage through up-regulation of tumor necrosis factor-alpha, inducible nitric oxide synthase, p53, c-myc, and bax-alpha, and suppression of bcl-2. J Cell Biochem 87: 266-278, 2002.

54. Sharif M, Whitehouse A, Sharman P, Perry M and Adams M: Increased apoptosis in human osteoarthritic cartilage corresponds to reduced cell density and expression of caspase-3. Arthritis Rheum 50: 507-515, 2004.

55. Loening AM, James IE, Levenston ME, et al: Injurious mechanical compression of bovine articular cartilage induces chondrocyte apoptosis. Arch Biochem Biophys 381: 205-212, 2000.

56. Cho YS: Perspectives on the therapeutic modulation of an alternative cell death, programmed necrosis (Review). Int J Mol Med 33: 1401-1406, 2014.

57. Liang W, Lin M, Li X, et al: Icariin promotes bone formation via the BMP-2/Smad4 signal transduction pathway in the hFOB 1.19 human osteoblastic cell line. Int J Mol Med 30: 889-895, 2012.

58. Zhang XM, Huang GW, Tian ZH, Ren DL and Wilson JX Folate stimulates ERK1/2 phosphorylation and cell proliferation in fetal neural stem cells. Nutr Neurosci 12: 226-232, 2009

59. Mebratu Y and Tesfaigzi Y: How ERK1/2 activation controls cell proliferation and cell death: Is subcellular localization the answer? Cell Cycle 8: 1168-1175, 2009. 
60. Imamichi Y, Waidmann O, Hein R, Eleftheriou P, Giehl K and Menke A: TGF beta-induced focal complex formation in epithelial cells is mediated by activated ERK and JNK MAP kinases and is independent of Smad4. Biol Chem 386: 225-236, 2005

61. Zuscik MJ, Hilton MJ, Zhang X, Chen D and O'Keefe RJ: Regulation of chondrogenesis and chondrocyte differentiation by stress. J Clin Invest 118: 429-438, 2008.

62. Chiquet M, Gelman L, Lutz R and Maier S: From mechanotransduction to extracellular matrix gene expression in fibroblasts. Biochim Biophys Acta 1793: 911-920, 2009.

63. Agarwal S, Deschner J, Long P, et al: Role of NF-kappaB transcription factors in antiinflammatory and proinflammatory actions of mechanical signals. Arthritis Rheum 50: 3541-3548, 2004.

64. Dossumbekova A, Anghelina M, Madhavan S, et al: Biomechanical signals inhibit IKK activity to attenuate NF-kappaB transcription activity in inflamed chondrocytes. Arthritis Rheum 56: 3284-3296, 2007.

65. Perera PM, Wypasek E, Madhavan S, et al: Mechanical signals control SOX-9, VEGF, and c-Myc expression and cell proliferation during inflammation via integrin-linked kinase, B-Raf, and ERK1/2-dependent signaling in articular chondrocytes. Arthritis Res Ther 12: R106, 2010.

66. Ferrara N: Vascular endothelial growth factor: basic science and clinical progress. Endocr Rev 25: 581-611, 2004.

67. Carlevaro MF, Cermelli S, Cancedda R and Descalzi Cancedda F: Vascular endothelial growth factor (VEGF) in cartilage neovascularization and chondrocyte differentiation: auto-paracrine role during endochondral bone formation. J Cell Sci 113: 59-69, 2000.
68. Chen C, Sun MZ, Liu S, et al: Smad4 mediates malignant behaviors of human ovarian carcinoma cell through the effect on expressions of E-cadherin, plasminogen activator inhibitor-1 and VEGF. BMB Rep 43: 554-560, 2010.

69. Schwarte-Waldhoff I and Schmiegel W: Smad4 transcriptional pathways and angiogenesis. Int J Gastrointest Cancer 31: 47-59, 2002.

70. Schwarte-Waldhoff I, Volpert OV, Bouck NP, et al: Smad4/ DPC4-mediated tumor suppression through suppression of angiogenesis. Proc Natl Acad Sci USA 97: 9624-9629, 2000.

71. Chowdhury TT, Bader DL and Lee DA: Dynamic compression counteracts IL-1 beta-induced release of nitric oxide and PGE2 by superficial zone chondrocytes cultured in agarose constructs. Osteoarthritis Cartilage 11: 688-696, 2003.

72. Nugent GE, Aneloski NM, Schmidt TA, Schumacher BL, Voegtline MS and Sah RL: Dynamic shear stimulation of bovine cartilage biosynthesis of proteoglycan 4. Arthritis Rheum 54: 1888-1896, 2006

73. Nam J, Aguda BD, Rath B and Agarwal S: Biomechanical thresholds regulate inflammation through the NF-kappaB pathway: experiments and modeling. PLoS One 4: e5262, 2009.

74. McNulty AL, Estes BT, Wilusz RE, Weinberg JB and Guilak F: Dynamic loading enhances integrative meniscal repair in the presence of interleukin-1. Osteoarthritis Cartilage 18: 830-838, 2010. 